\section{RMD
Open}

Rheumatic \&

Musculoskeletal Diseases

To cite: Hua C, Buttgereit $F$, Combe B. Glucocorticoids in rheumatoid arthritis: current status and future studies. RMD Open 2020;6:e000536. doi:10.1136/ rmdopen-2017-000536

Received 27 June 2019 Revised 5 November 2019 Accepted 13 November 2019

\title{
Glucocorticoids in rheumatoid arthritis: current status and future studies
}

\author{
Charlotte Hua (ㄷ, , ${ }^{1}$ Frank Buttgereit $(1),{ }^{2}$ Bernard Combe (i) ${ }^{3}$
}

\section{ABSTRACT}

Since their first use for treating rheumatoid arthritis (RA) in the late 1940s, glucocorticoids (GCs) have been representing a substantial part of the therapeutic arsenal for RA. However, even if GCs are still widely prescribed drugs, their toxicity is discussed controversially, so obtaining consensus on their use in RA is difficult. Hence, the most recent European League Against Rheumatism and American College of Rheumatology recommendations on early arthritis and RA management advocate the use of GCs as adjunct treatment to conventional synthetic disease-modifying antirheumatic drugs, at the lowest dose possible and for the shortest time possible. However, the recommendations remain relatively vague on dose regimens and routes of administration. Here, we describe literature data on which the current recommendations are based as well as data from recent trials published since the drafting of the guidelines. Moreover, we make proposals for daily practice and provide suggestions for studies that could help clarifying the place of GCs in RA management. Indeed, numerous items, including the benefit/risk ratio of low-dose and very low-dose GCs and optimal duration of GCs as bridging therapy, remain on the research agenda, and future studies are needed to guide the next recommendations for RA.

\section{INTRODUCTION}

Check for updates

(c) Author(s) (or their employer(s)) 2020. Re-use permitted under CC BY-NC. No commercial re-use. See rights and permissions. Published by BMJ.

${ }^{1}$ Rheumatology Department, Nîmes Hospital, EA2415, Montpellier University, Nîmes, France

${ }^{2}$ Department of Rheumatology and Clinical Immunology, Charite University Medicine Berlin (CCM), Berlin, Germany ${ }^{3}$ Rheumatology Department, Montpellier hospital, UMR 5535, Montpellier University, Montpellier, France

Correspondence to Dr Charlotte Hua; hua.charlotte@gmail.com
In this time of targeted therapies, therapeutic strategies and management of comorbidities in patients with rheumatoid arthritis (RA), the question of the future position glucocorticoids (GCs) may have in RA is worth asking. Indeed, although therapy with GCs was a major therapeutic advance in the $1950 \mathrm{~s}$ for RA, the current emphasis is more on the disadvantages than the benefits of this treatment. However, in light of recent literature data, low-dose GCs still seem to have an important role in RA. ${ }^{1-3}$

The goal of treatments for patients with $\mathrm{RA}$ in the short term is to decrease disease activity and achieve clinical remission, and in the medium term to limit or prevent structural progression, disability and systemic manifestations. Available disease-modifying antirheumatic drugs (DMARDs) have shown their efficacy. Conventional synthetic (cs)

\section{Key messages}

What is already known about this subject?

- Glucocorticoids are effective drugs in rheumatoid arthritis (RA), but because of their toxicity, their use requires care and vigilance.

What does this study add?

- This review presents and discusses recent literature data on glucocorticoids use in RA and provides suggestions for future research in the field of glucocorticoids in RA.

How might this impact on clinical practice?

- Glucocorticoids in RA must be used in combination with disease-modifying antirheumatic drugs (DMARDs), notably as bridging therapy with conventional synthetic DMARDs.

- Evaluation of the benefit/risk ratio must be systematic, even for low-dose glucocorticoids.

DMARDs have a relatively long onset of action while most of biological (b) and targeted synthetic (ts) DMARDs (bDMARDs and tsDMARDs) act faster. GCs in RA provide the advantage of a rapid onset of action, which allows waiting for the onset of csDMARDs efficacy. Moreover, even if GCs are mainly widely used whenever clinicians need rapid symptomatic relief for their patients with RA, their structural effect must not be forgotten. However, the benefit/risk ratio of GCs remains precarious and their modalities of use in RA remain controversial.

In this review, we first detail the latest European and US recommendations on GCs use in RA and discuss the use of GCs in current practice. Then, we consider recent literature data on GCs efficacy (both clinical and structural) in RA and the various ways of using GCs. Finally, we address the adverse effects potentially associated with GCs. Our work being a narrative review and not a systematic review, we have not included all the existing studies but only the ones seeming the most relevant to us. Moreover, during this review, 
we propose topics that could be of interest to be evaluated in future studies of GCs use in RA.

\section{INTERNATIONAL RECOMMENDATIONS European recommendations}

Recent updates of the European recommendations for management of early arthritis and $\mathrm{RA}^{4-6}$ placed a greater focus on the benefit of GCs therapy than the previous versions. Short-term GCs therapy should now be considered as part of the initial treatment strategy and subsequently if an initial strategy has failed, as bridging therapy if a change in a csDMARD is considered. ${ }^{6}$ GCs should be tapered as rapidly as is clinically feasible: long-term use of GCs should be avoided, GCs should be gradually reduced and stopped, usually within 3 months and only exceptionally by 6 months. ${ }^{6}$ The term 'low-dose' GCs was replaced by 'short-term' GCs to take into account several current ways of using GCs, such as parenteral bolus. ${ }^{6}$

\section{US RECOMMENDATIONS}

The 2015 American College of Rheumatology (ACR) guidelines for early and established RA recommend adding GCs to DMARDs during disease flares, at the lowest dose and for the shortest period possible. ${ }^{7}$ In contrast to the European recommendations, adding GCs when a csDMARD is initiated depends on disease activity.

Taken together, these international recommendations agree on the use of GCs for disease flare and possibly at the start of a new csDMARD. Specific advice concerning dosage, duration, route of administration and strategies is limited and less consensual, because reliable and detailed evidence is scarce. For US recommendations, a dose $<10 \mathrm{mg} /$ day is considered a low dose, and GCs should be tapered in less than 3 months, whereas for European recommendations, the threshold is $7.5 \mathrm{mg}$ /day, and GCs could be prescribed in combination with csDMARDs for up to 6 months maximum, knowing that this duration is mainly expert-driven. ${ }^{8}$ Despite these differences, international guidelines underline the importance of GCs but also advocate the use of GCs at the lowest cumulative dose possible because of the high awareness of potentially associated adverse effects.

In these sets of recommendations as well as in this review, GCs dosages are expressed in prednisone equivalent.

\section{CURRENT PRACTICE}

A recent study of an Australian cohort of patients with RA showed that the probability of GCs use throughout follow-up has decreased over time, from $55 \%$ in 2001 to $39 \%$ in $2012 \quad(\mathrm{p}<0.001) .{ }^{9}$ In this cohort, current csDMARD use but not bDMARD use was associated with increased current GCs use. In a recent analysis considering the years from 1980 up to 2004, the reduction of mean initial low-dose, for long-term GCs therapy in $\mathrm{RA}$, was found from $10.3 \mathrm{mg} /$ day up to $3.6 \mathrm{mg} /$ day. ${ }^{10} \mathrm{In}$ contrast, another observational cohort study showed that the proportion of patients initiating GCs was higher in the group from 1995 to 2007 compared with the earlier group from 1980 to 1994 (68\% vs $36 \%$ ) but the cumulative dose did not differ over the first year. ${ }^{11}$

Anyway, GCs are still widely used in RA. GCs appear to be used in approximately $50 \%$ of patients with RA, ${ }^{12}$ with varied duration and dosage among the studies. In the German CAPEA cohort of patients with early arthritis, $82 \%$ received methotrexate (MTX) within the first months, $77 \%$ received GCs and 20\% of these received $<7.5 \mathrm{mg}$ /day prednisone but one third received $>20 \mathrm{mg}$ / day. ${ }^{13}$ After 2 years of follow-up, $12 \%$ of the patients received biologics, $52 \%$ were free of GCs and $41 \%$ were receiving $<5 \mathrm{mg}$ /day. In the French ESPOIR cohort of patients with early arthritis, $45 \%$ started GCs during the first 6 months and more than $50 \%$ received GCs at least once over 5 years after inclusion. ${ }^{14}$ Overall, the dose of GCs received during follow-up was very low, the mean was $3.1 \pm 2.9 \mathrm{mg} /$ day ${ }^{15}$ In the Canadian CATCH cohort of patients with RA, $42 \%$ were considered GCs users and the median oral daily dose was $5 \mathrm{mg}$ (IQR 2.5-10). ${ }^{16}$

\section{GLUCOCORTICOIDS EFFICACY \\ Clinical efficacy \\ Current knowledge}

For reasons of brevity, in this part, we will focus on only the most relevant and recent data on clinical efficacy of GCs in RA published during the last 6 years (table 1). In the following parts of our work, we will discuss other relevant studies published earlier.

Overall, these results agreed with previous findings and with the conclusion of the most recent systematic literature reviews published on this topic and showed a beneficial effect of GCs when added to csDMARDs. ${ }^{5} 17$ The available data primarily relate to GCs in addition to csDMARDs and not, or not specifically, to bDMARDs or tsDMARDs. Moreover, the current literature concerns mainly patients with early arthritis, and studies reporting on GCs efficacy in patients with established RA are clearly less frequent.

In the CAPRA-2 trial, a study of patients with established RA (disease duration approximately 8 years) and active disease, low-dose $(5 \mathrm{mg} /$ day $)$ prednisone with a modified release formulation (chronotherapy) added to existing DMARDs significantly ameliorated disease activity at 12 weeks as compared with placebo (PBO) added to existing DMARDs. ${ }^{18} 19$ DMARDs were almost exclusively csDMARDs because only one patient in each group received a bDMARD.

In the CareRA trial, patients with early RA without poor prognosis markers were randomised to one of two treatment arms. ${ }^{20}$ In one arm, GCs were initially associated with MTX $(30 \mathrm{mg} /$ day prednisone decreased to $5 \mathrm{mg}$ / day in 6 weeks) while in the other arm, MTX was initiated without GCs. Disease Activity Score in 28 joints (DAS28) remission at 16 weeks was more frequently achieved in 
Table 1 Characteristics of studies of the clinical efficacy of glucocorticoids published in the last 6 years

\begin{tabular}{|c|c|c|c|c|c|}
\hline Study & $\begin{array}{l}\text { Disease } \\
\text { duration }\end{array}$ & Intervention & $\begin{array}{l}\text { Period of } \\
\text { evaluation }\end{array}$ & Outcomes & Results \\
\hline $\begin{array}{l}\text { Buttgereit et al } \\
(\text { CAPRA-2) })^{15}\end{array}$ & Mean 8 years & $\begin{array}{l}\text { MR prednisone ( } 5 \mathrm{mg} / \text { day) (Gp 1) or } \\
\text { PBO (Gp 2)+existing DMARDs }\end{array}$ & 12 weeks & ACR 20 & $\begin{array}{l}\text { Gp 1: } 48 \% \\
\text { Gp 2: } 29 \% *\end{array}$ \\
\hline $\begin{array}{l}\text { Verschueren et al } \\
(\text { CareRA })^{20}\end{array}$ & $\leq 1$ year & $\begin{array}{l}\text { csDMARDs with (Gp 1) or w/o (Gp 2) } \\
\text { GCs ( } 30 \mathrm{mg} / \text { day to } 5 \mathrm{mg} \text { /day in } 6 \text { weeks) }\end{array}$ & 16 weeks & DAS28-CRP<2.6 & $\begin{array}{l}\text { Gp 1: } 65 \% \\
\text { Gp 2: } 47 \%\end{array}$ \\
\hline 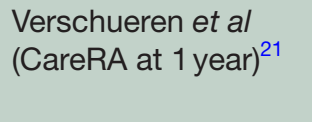 & $\leq 1$ year & $\begin{array}{l}\text { csDMARDs with (Gp 1) or w/o (Gp } \\
\text { 2) GCs ( } 30 \mathrm{mg} / \text { days to } 5 \mathrm{mg} / \text { days in } \\
6 \text { weeks) }\end{array}$ & 1 year & DAS28-CRP<2.6 & $\begin{array}{l}\text { Gp 1: } 67 \% \\
\text { Gp 2: } 57 \%\end{array}$ \\
\hline $\begin{array}{l}\text { Markusse et al (BeSt } \\
\text { at 10years) }\end{array}$ & $\leq 2$ years & $\begin{array}{l}\text { Initial groups of randomisation: MTX } \\
\text { then substituted with csDMARDs (Gp 1) } \\
\text { or MTX then addition of csDMARDs (Gp } \\
\text { 2) or COBRA scheme=MTX+SSZ+GCs } \\
\text { (60 mg/day to } 7.5 \text { mg/day in } 6 \text { weeks) } \\
\text { (Gp 3) or MTX+IFX (Gp 4) }\end{array}$ & 10 years & DAS44 <1.6 & $\begin{array}{l}\text { Approx. } 50 \% \\
\text { in each Gp }\end{array}$ \\
\hline $\begin{array}{l}\text { Safy et al (CAMERA- } \\
\text { II follow-up) }\end{array}$ & $\leq 1$ year & $\begin{array}{l}\text { Initial groups of randomisation: GCs } \\
\text { (10 mg/day) (Gp 1) or PBO (Gp 2)+MTX }\end{array}$ & $\begin{array}{l}\text { Median } \\
6.6 \text { years }\end{array}$ & $\begin{array}{l}\text { Initiation of first } \\
\text { bDMARD }\end{array}$ & $\begin{array}{l}\text { Gp 1: } 31 \% \\
\text { Gp 2: } 50 \% *\end{array}$ \\
\hline $\begin{array}{l}\text { Ajeganova et } \\
\text { al (BARFOT at } \\
10 \text { years) }\end{array}$ & $\leq 1$ year & $\begin{array}{l}\text { Initial groups of randomisation: } \\
\text { csDMARDs with (Gp 1) or w/o (Gp 2) } \\
\text { GCs (7.5 mg/day) }\end{array}$ & 10years & Use of bDMARD & $\begin{array}{l}\text { Gp 1: } 15 \% \\
\text { Gp 2: } 15 \%\end{array}$ \\
\hline
\end{tabular}

${ }^{*} \mathrm{P}<0.05$

ACR, American College of Rheumatology; approx, approximately;bDMARD, biological disease modifying antirheumatic drugs; CRP, C reactive protein; csDMARDs, conventional synthetic disease modifying antirheumatic drugs; DAS28, Disease Activity Score in 28 joints; DAS44, disease activity score in 44 joints; GCs, glucocorticoids; Gp, group; MR, modified release; MTX, methotrexate; PBO, placebo; SSZ, sulfasalazine; w/o, without.

patients with than without GCs, although not significantly $(65 \%$ vs $47 \%, \mathrm{p}=0.08) .{ }^{20}$ According to data at 1 and 2 years, the rates of remission were still higher in the MTX+GCs than MTX-only arm but again not significantly. ${ }^{21} 22$

Ten-year data from the BeSt study were published in $2016 .{ }^{23}$ In this trial, 508 patients with early active RA were randomised to four arms: a pre-established maintenance treatment regimen prescribed as monotherapy beginning with MTX; a step-up group with sulfasalazine (SSZ) added to MTX in case of failure; a group following the COBRA scheme (SSZ, MTX and GCs initially at $60 \mathrm{mg}$ /day, then progressively tapered to $7.5 \mathrm{mg} /$ day in 6 weeks) and a group receiving MTX and infliximab from the beginning. ${ }^{24}$ In the initial study, patients following the COBRA strategy showed better clinical efficacy at 3 months than patients without GCs. At 10 years, approximately $50 \%$ of patients were in remission whatever their initial group of randomisation. In the meantime, strategy-driven changes have occurred and it is thus difficult to conclude on the long-term effect of initial GCs therapy. ${ }^{23}$

Results of the post-trial follow-up of the CAMERA-II study were published in $2017 .^{25}$ In this study of 236 patients with early RA, the addition during 2 years of $10 \mathrm{mg} /$ day prednisone to MTX was compared with a MTX and PBO group. ${ }^{26}$ Disease activity after 2 years of treatment improved more on average in the MTX-GCs arm than MTX-PBO arm, but the differences observed in the first months tended to decrease over time. In the follow-up study (median follow-up of approximately 6.6 years), significantly fewer patients of the MTX-GCs group had initiated a bDMARD than those from the MTX-PBO group $(31 \%$ vs $50 \%, \mathrm{p}<0.05) .^{25}$

Data at 10 years from the BARFOT trial have been reported. ${ }^{27}$ In this open randomised trial of 250 patients with early RA, the addition during 2 years of $7.5 \mathrm{mg}$ / day prednisolone to csDMARDs was compared with csDMARDs alone. Clinical outcomes were better in the GCs group at all time points $(3,6,12,18$ and 24 months). ${ }^{28} \mathrm{~A}$ follow-up study at 4 years no longer found differences in the proportion of patients in remission between the groups. ${ }^{29}$ At 10 years, bDMARD use did not differ with and without GCs. ${ }^{27}$ Of note, the proportion of patients who used a bDMARD was very low (15\% in each group), perhaps because patients were included in the BARFOT cohort between 1995 and 1999, before the era of biologics.

In light of these and other literature data, there is little doubt that GCs are effective for reducing disease activity in patients with RA, at least in the short term. However, confirming whether the clinical benefit of GCs persists in the medium and long term is difficult. Because of their toxicity and moderate structural effect (see below), GCs cannot be considered monotherapy and should always be used together with DMARDs.

\section{Studies to perform}

We notably lack studies evaluating the clinical effect in the medium and long term with short-term $(<6$ months) GCs therapy. Such studies should be RCTs including 
patients with RA for which a specific DMARD has to be initiated. These patients should be randomised into an arm receiving the DMARD with GCs discontinued over 3 months and an arm receiving the DMARD without GCs. Clinical efficacy measures should occur at medium and long term: after several months and even years of follow-up. As stated above, it would be of particular interest to conduct such studies in patients initiating a targeted DMARD and not only in patients initiating a csDMARD. Trials addressing the advantages provided by new formulations of GCs would also be of interest. Indeed, the pharmaceutical field of GCs continues to evolve, notably with the development of modified-release or delayed-release prednisone (chronotherapy) or GC receptor agonists to improve the efficacy and/or reduce toxicity. ${ }^{30-32}$ Modified night-release formulation of lowdose prednisone, although administered in the evening (acting like a replacement therapy), has been developed to contrast the circadian rise in proinflammatory cytokine levels (night), that contributes to RA disease activity and might represent the way to further optimise the DMARD activity exerted by low dose GCs in RA. ${ }^{33}$

\section{Structural effect}

Current knowledge

In this part, we have included all the studies on structural effects of GCs seeming relevant to us, without any time limit. In most trials, GCs were used in combination with csDMARDs, so concluding on the specific effect of GCs is difficult.

In 2005, the BARFOT randomised trial showed the slowing at 2 years of the structural progression more important with than without GCs $(0.2 \%$ vs $0.4 \%$, $\mathrm{p}=0.02) .{ }^{28}$ The follow-up at 4 years suggested a lower increase in total Sharp score during the 4 years with than without GCs, although not significantly $(\mathrm{p}=0.079){ }^{29}$ In 2007, a meta-analysis of 15 studies was in favour of a significant reduction of erosion progression by GCs given in addition to csDMARDs. ${ }^{34}$ In 2010, the structural impact of GCs added to csDMARDs was evaluated in another meta-analysis, showing approximately $70 \%$ reduced radiographic progression $(\mathrm{p}=0.0008) .{ }^{35}$ In this meta-analysis, the combination of GCs to csDMARDs had a similar effect as bDMARDs added to MTX (difference of $7 \%$ in radiographic progression between the two groups, $\mathrm{p}=0.44$ ). However, the conclusions of this meta-analysis were limited by the high heterogeneity of included trials. In the 11-year follow-up study of the COBRA trial, structural damage progression was lower in the group initially treated according to the COBRA scheme (MTX+SSZ+GCs $(60 \mathrm{mg} /$ day to $7.5 \mathrm{mg} /$ day in 6 weeks $))$ than in the group initially treated with SSZ monotherapy. ${ }^{36}$

More recently, in the CAMERA-II study, radiographic progression with GCs was significantly reduced, with $78 \%$ of patients in the MTX-GCs group remaining erosion-free after 2 years vs only $67 \%$ in the MTX-PBO group. ${ }^{26}$ Similarly, at the 2-year post-trial follow-up, the median erosion score was significantly lower in the former MTX-GCs group than in the former MTX-PBO group $(\mathrm{p}=0.002){ }^{25}$

In light of the literature, the structural effect of GCs seems clear, at least when GCs are added to csDMARDs. Moreover, this positive effect on radiographic progression seems to last even after discontinuation of GCs. Obviously, when combined with bDMARDs or tsDMARDs, showing any additional effect of GCs is difficult because these drugs already strongly inhibit structural progression.

\section{Studies to perform}

Studies comparing different durations of GCs treatment as bridging therapy in terms of structural protection are needed to increase the strength of the recommendations concerning the optimal period of GCs prescription. The approach of these studies would be to evaluate if a shortterm ( $<6$ months) GCs therapy added to MTX is as effective as a more prolonged GCs therapy (during 2 years, eg, as in CAMERA-II or BARFOT trials) on structural progression at medium and long term.

\section{GLUCOCORTICOIDS USE STRATEGIES}

For this part, selection of the studies was based only on their relevance and not on their publication date.

\section{Bridging therapy}

Current knowledge

When and how to introduce and taper GCs are important questions for rheumatologists and their patients. In view of the risks and benefits, the usual practice is to use an induction strategy followed by a progressive decrease. This is notably done when GCs are prescribed as bridging therapy along with the introduction of a csDMARD, to wait for its efficacy. This scheme was used in the COBRA study, with results published in $1997 .{ }^{37}$ In this study, 155 patients with early RA were randomised into two arms: combination treatment with SSZ, MTX and prednisolone (initially at $60 \mathrm{mg} /$ day, then progressively tapered to $7.5 \mathrm{mg}$ /day in 6 weeks) compared with SSZ only. The results favoured the GCs arm, both on the clinical and on the structural effects. Since this study, various strategies for the use of GCs have been evaluated, but few RCTs have been designed to specifically compare dosage, duration and tapering of GCs.

Recently, in two articles reporting on the same RCT, two different GCs strategies were compared: the COBRAlight strategy (prednisolone at $30 \mathrm{mg} /$ day, tapered to $7.5 \mathrm{mg} /$ day within 9 weeks) combined with MTX, and the original COBRA strategy. ${ }^{38} 39$ The outcomes were similar between the two arms in terms of reducing clinical disease activity, improving functional ability and preventing radiographic progression, showing that treatment regimens including medium-dose GCs are not inferior to regimens with initially high-dose GCs. ${ }^{40}$

A posthoc analysis of six phase III studies evaluating tofacitinib in RA, in monotherapy (ORAL start, ORAL Solo) or combined with csDMARDs (ORAL Scan, ORAL Standard, ORAL Sync, ORAL Step), examined the 
impact of the presence of GCs in the treatment groups. ${ }^{41}$ Mean dose of GCs was approximately of $6 \mathrm{mg}$ /day. Across all studies, the concomitant use of GCs did not affect the clinical efficacy of tofacitinib. In contrast, response was frequently better for patients receiving MTX with GCs than without GCs, but the differences were often not statistically significant.

Moreover, in an analysis presented at the 2018 ACR meeting but not yet published of pooled data from four RCTs (AMBITION, ACT-RAY, ADACTA and FUNCTION) on intravenous tocilizumab (TCZ), concomitant GC therapy had no impact on the clinical efficacy of TCZ at 24 weeks. ${ }^{42}$ The authors assessed patients in the comparator arms: the clinical response of patients receiving adalimumab as well as those initiating MTX was not affected by the use of GCs. Data on the mean GCs dose were lacking in this abstract.

According to a posthoc analysis of the TOZURA trial, a phase IV study evaluating subcutaneous TCZ, the proportion of patients achieving DAS28 remission at 24 weeks was broadly similar between the groups receiving TCZ, with or without GCs (median dose $5 \mathrm{mg} /$ day) ${ }^{43}$

However, concerning the quoted studies on tofacitinib and TCZ, it is necessary to specify that GCs use was not submitted to randomisation and could be prior to the beginning of the study making it difficult to compare the groups with and without GCs.

\section{Studies to perform}

The current literature mainly concerns the use of GCs as bridging therapy when a csDMARD is initiated, but we have few studies on the use of GCs as bridging therapy when a bDMARD or tsDMARD is initiated. Indeed, the onset of action might be faster for bDMARDs, and even more so for tsDMARDs, than csDMARDs, which could explain why the recent posthoc analyses of the tofacitinib and TCZ studies did not find a benefit of adding GCs. It would be useful to have RCTs designed to evaluate clinical and structural efficacy of a short-term GCs therapy prescribed when a targeted DMARD is initiated. Finally, studies on the best initiation dosage of GCs are lacking, as are those on the duration of GCs as bridging therapy and trials comparing several tapering strategies.

\section{Flare treatment}

\section{Current knowledge}

Data on this topic are scarce, among other reasons due to lack of an agreed-upon definition of RA flare. ${ }^{44}$ In an older study of 18 patients with RA, flares were treated with GCs (prednisone $25 \mathrm{mg}$ /day tapered in 5 days) or PBO. ${ }^{45}$ Clinical outcomes were better at 6 months when GCs were used. In the BELIRA trial, patients with RA with active disease received a total of $250 \mathrm{mg}$ /day prednisolone over 1 week added to their existing csDMARDs. ${ }^{46}$ This strategy resulted in a highly significant improvement in clinical, functional and serological measures at 1 week. A RCT comparing the efficacy of three intravenous pulses of $120 \mathrm{mg} /$ day dexamethasone or $1 \mathrm{~g} /$ day methylprednisolone suggested that the two strategies were safe and effective for RA flares at 1 month. ${ }^{47}$

Studies to perform

Daily management of RA would be helped by studies investigating the modalities of treating disease flares with GCs, for example, by comparing pulse therapy to lowdose oral GCs.

\section{Maintenance therapy \\ Current knowledge}

Despite recommendations, many patients are already being treated for months and years with low doses GCs, without apparent excessive toxicity, and there is no consensus on whether the GCs therapy should be stopped or not. In the international, multicentre SEMIRA trial, 259 patients with RA in remission and treated for more than 6 months by TCZ \pm csDMARDs+GCs were randomly assigned to continued GCs or GCs taper. ${ }^{48}$ Even if continuing GCs provided better disease control than tapering GCs, almost two-thirds of patients still achieved treatment success at 24 weeks and could stop GCs entirely.

\section{Studies to perform}

Results of this trial are suggesting that tapering and discontinuation of GCs should be tested in patients in remission under TCZ and receiving long-term low-dose GCs. In case of a flare, reintroduction of GCs should be considered. Similar studies on patients with RA receiving other treatments than TCZ would be of interest in order to extrapolate the results.

\section{OTHER ADMINISTRATION ROUTES \\ Parenteral route \\ Current knowledge}

In a study published in 1993, 41 patients with RA were randomised to receive methylprednisolone orally $(500 \mathrm{mg})$ or intramuscularly $(120 \mathrm{mg})$ at baseline and 4 and 8 weeks. ${ }^{49}$ At 16 weeks, the intramuscular route seemed better than the oral administration on pain and Health Assessment Questionnaire (HAQ) score. In the tREACH trial, patients with early RA received csDMARDs (MTX monotherapy or MTX+SSZ+hydroxychloroquine (HCQ) ) and oral GCs started at $15 \mathrm{mg} /$ day and tapered during 10 weeks or MTX+SSZ+HCQ plus an initial intramuscular pulse of GCs. ${ }^{50}$ The two groups did not differ at 1 year in clinical response, structural progression or safety.

\section{Studies to perform}

From the literature data, concluding on the superiority of one GCs route of administration versus another is difficult and that is why current recommendations state that different dose regimens and route of administration can be used. ${ }^{6}$ Strategy trials, with designs similar to that of the tREACH study, comparing the efficacy of one or several parenteral (intravenous or intramuscular) GCs injections versus initiation and tapering of oral GCs as 
bridging therapy could answer this question. Besides efficacy issues, the parenteral form could be beneficial for preventing self-medication by patients and avoiding the risk with GCs tapering and mid-term to long-term GCs use. $^{51}$

\section{Intra-articular injections}

Current knowledge

Subanalyses of the BeSt study showed that 3 months after intra-articular GCs injection, $50 \%$ of joints were no longer swollen. After 1 year, swelling had recurred in $14 \%$ of joints with initially good clinical response. ${ }^{52}$ Initially, disease activity scores significantly improved, but over time, DAS and HAQ scores became similar in injected and non-injected patients. After 8 years, the two groups did not differ in joint damage. ${ }^{40}$ In the CIMESTRA trial, patients with early RA received intra-articular GCs in any swollen joint (maximum four joints per visit) combined with step-up csDMARDs treatment over 2 years. ${ }^{53}$ Oral GCs were not allowed. At 2 years, the median cumulative number of injections for each patient was 13, and the cumulative dose of GCs was $<1 \mathrm{mg} /$ day. The injections had a rapid-onset anti-inflammatory action, and 2 weeks after inclusion, $39 \%$ of patients were in DAS28 remission. At the end of follow-up, $55.5 \%$ of joints injected at baseline did not show disease relapse, and intra-articular injections were well tolerated.

Recently, one small and open-label RCT showed greater clinical efficacy (ACR20/50/70 response) of csDMARDs combined with initial intra-articular GCs injections than csDMARDs alone in patients with early RA without oral GCs. ${ }^{54}$

\section{Studies to perform}

Larger RCTs with a design similar to the trial by Menon et $a b^{4}$ and with compliance to blinding are needed, as are RCTs comparing intra-articular GCs injections with other routes of administration.

\section{GLUCOCORTICOIDS SAFETY}

In this part also, the publication date was not taken into account for the selection of the studies. Due to the considerable amount of data published on this topic, we have decided to notably quote meta-analysis and literature reviews.

\section{Current knowledge}

GCs toxicity is a major concern when higher dosages are given for a longer time. However, even low-dose to medium-dose GCs are associated with adverse effects.

In 2007, a EULAR taskforce published recommendations for the management of systemic GCs therapy in rheumatic diseases. ${ }^{55}$ According to a literature review, the taskforce identified the following main adverse events of GCs: cardiovascular diseases, infections, gastrointestinal diseases, psychological disorders, endocrine pathologies, dermatological issues, musculoskeletal disorders (including osteoporosis) and ophthalmological diseases. ${ }^{55}$

In a meta-analysis of trials and follow-up studies published in 2009, the rate of adverse effects linked to GCs (prescribed for RA) at $<30 \mathrm{mg} /$ day was $43 / 100$ patient-years (95\% CI 30 to 55). ${ }^{56}$ Another meta-analysis, also published in 2009, pooled the results of six RCTs comparing low-dose GCs (mean dose $5-10 \mathrm{mg}$ /day) to PBO ${ }^{57}$ According to this meta-analysis, the groups did not differ in any type of event or serious adverse events. According to the 11-year follow-up study of the COBRA trial, rates of hypertension and diabetes were higher in the group initially treated according to the COBRA scheme than in the group initially treated with SSZ monotherapy but rates of cardiovascular disease were similar between the two groups, knowing that patients from this group had also received GCs during follow-up. ${ }^{36}$ After 23 years of follow-up, the mortality rate of patients from the COBRA trial was similar to that of the general population, whatever the initial randomisation group. ${ }^{58}$ From the 10-year data of the BARFOT trial, incident cardiovascular events were evenly distributed with and without GCs ( $15 \%$ and $14 \%)$, but the risk of the first cerebrovascular event was almost four times higher with than without GCs (HR 3.7 (95\% CI 1.2 to 11.4), with higher mortality with GCs but not significantly. ${ }^{27}$ Of note, $53 \%$ of the patients with GCs had stopped them after the first 2 years, and the mean dose of prednisolone for those taking GCs was $7.2 \pm 1.1 \mathrm{mg} /$ day at the 2-year follow-up, $6.5 \pm 3.6 \mathrm{mg} /$ day at 4 years and $4.9 \pm 3.3 \mathrm{mg} /$ day at 8 years. ${ }^{27}$ Long-term (median follow-up almost 7 years) post-trial data from the CAMERA-II study are in agreement with these findings, showing no statistical difference in incident GCs-related comorbidities between the MTX-GCs and MTX-PBOarms, but numerical differences in cardiovascular events (13 vs 8$)$ and death (10 vs 6) ${ }^{25}$ Similar to the BARFOT post-trial study, the controlled design was stopped after the first 2 years, and only half of the patients from the MTX-GCs arm had stopped GCs at 3 years. In summary, data from RCTs are quite reassuring in terms of low-dose GCs safety, but most RCTs had only a short follow-up. However, in the two long-term post-trial studies of BARFOT and CAMERA-II, in which patients had initially taken GCs during 2 years, GCs were associated with more cardiovascular events, but not significantly.

Observational studies usually showed significantly more adverse events in patients with than without GCs, whether for cardiovascular events or for other adverse effects linked to GCs such as infections ${ }^{59}$ or osteoporosis, and even for low-dose GCs. ${ }^{60}$ However, data from observational studies should be considered with caution, notably because of population heterogeneity and confounding by indication. Nevertheless, cardiovascular toxicity with low-dose GCs was confirmed in a recent meta-analysis of RCTs and observational studies, noting $47 \%$ increased cardiovascular events in patients with RA receiving GCs. ${ }^{61}$ In 2014, a North American study reported a threshold of $8-15 \mathrm{mg} /$ day for increased mortality linked to GCs 
in RA (adjusted HR 1.78 (95\% CI 1.22 to 3.60)), after adjusting for potential confounders and the propensity to receive GCs. ${ }^{62}$ The minimum total cumulative dose associated with all-cause mortality was $40 \mathrm{~g}$ (adjusted HR 1.89 (95\% CI 1.25 to 2.44$)$ ). ${ }^{62}$ Reaching this cumulative dose threshold would take approximately 21 years with a daily dose of $5 \mathrm{mg}$ /day. Patients with RA can commonly reach such a duration of exposure. ${ }^{63}$

It is clear that adverse effects are related to the dose and duration of GCs but the data on the toxic effects of a low cumulative dose of GCs are scarce. In the CareRA trial, the proportion of patients with adverse events at 1 year was similar between patients who had received GCs according to the COBRA Slim scheme and patients without GCs. ${ }^{21}$ A recent study based on data from the French cohort of early arthritis patients (ESPOIR cohort) found no significant over-risk at 7 years of follow-up, despite numerical differences in cardiovascular events, infectious diseases or osteoporotic fracture between patients who received very low-dose GCs (mean dose $3.1 \pm 2.9 \mathrm{mg} /$ day) and patients who never received GCs. ${ }^{15}$

\section{Studies to perform}

Studies comparing various strategies of GCs use in terms of the frequency of adverse effects could help practitioners to prescribe GCs in the most suitable way. The toxic effects of GCs are clearly dose-dependent but are also influenced by individual factors, and studies evaluating the toxicity of GCs should take into account the comorbidities of the patients with RA to whom GCs are prescribed so as to identify the patients for whom the risk/benefit ratio is favourable or not.

\section{CONCLUSION}

GCs clearly still have an important role to play in RA management. Their clinical (and structural) efficacy is widely acknowledged. Most studies have evaluated GCs efficacy as bridging therapy, combined with csDMARDs. Consequently, available studies have mainly focused on patients with early RA, and data for established RA are scarce. Nevertheless, GCs seem useful in this population to control flares. By contrast, GCs monotherapy does not represent an acceptable therapeutic option. The benefit of adding GCs to bDMARDs and tsDMARDs is most likely low or non-existent because of the fast onset of action of most of these DMARDs. GCs are also cheap, and their combination with csDMARDs could reduce or delay the use of bDMARDs or tsDMARDs.

However, there is also evidence of GCs toxic effects, notably with moderate-dose to high-dose GCs used for a longer time, but even low-dose GCs might have adverse effects. Besides, the long-term GCs safety might be linked to the cumulative dose, and, apart from the daily dose, the duration is also crucial. GCs must be used at the lowest possible dose and for the shortest possible time. With long-term ( $\geq 6$ months) GCs therapy, EULAR has defined $5 \mathrm{mg} /$ day or less (if needed for controlling the disease) as the acceptable daily intake in terms of cardiovascular risk and risk of hyperglycaemia/diabetes, osteoporosis and infection for the vast majority of patients, but the individual risk of harm must also be evaluated by taking into account patient-specific characteristics. ${ }^{64}$ The ongoing trial GLORIA, a 2-year pragmatic RCT aiming to assess the safety and efficacy of GCs at $5 \mathrm{mg}$ /day vs PBO added to DMARDs in elderly patients with RA ( $\geq 65$ years), could provide evidence to support further this threshold of $5 \mathrm{mg} /$ day. ${ }^{65}$ In the same prospects of improving the risk/benefit ratio of GCs, studies of chronotherapy and GCs agonists seem promising. ${ }^{31} 32$

Current recommendations do not advocate a specific administration route versus another. Indeed, according to the available literature data, oral and parenteral use of GCs seem to have similar efficacy, but data are scarce and do not concern structural efficacy. However, the parenteral form might facilitate GCs withdrawal by preventing self-medication. Each GCs prescription must be preceded by an evaluation of the benefit/risk balance and by information provided to the patient in the context of a shared decision.

New studies with modern designs evaluating GCs in RA are still needed. Considering the existing literature, we have proposed in this review several leads to guide future research. Of note, future trials will also need to include cost-utility analyses and data on patient participation. ${ }^{66}$ In continuum with these future studies, further guideline updates will need to address specific conditions and circumstances for which GCs should be prescribed in order to improve the balance between efficacy and longterm safety.

Contributors Conception or design of the work: $\mathrm{CH}, \mathrm{BC}$. Drafting the article: $\mathrm{CH}, \mathrm{BC}$. Critical revision of the article: $\mathrm{BC}$, FB. Final approval of the version to be published: $\mathrm{CH}, \mathrm{FB}, \mathrm{BC}$.

Funding The authors have not declared a specific grant for this research from any funding agency in the public, commercial or not-for-profit sectors.

Competing interests None declared.

Patient consent for publication Not required.

Provenance and peer review Commissioned; externally peer reviewed.

Open access This is an open access article distributed in accordance with the Creative Commons Attribution Non Commercial (CC BY-NC 4.0) license, which permits others to distribute, remix, adapt, build upon this work non-commercially, and license their derivative works on different terms, provided the original work is properly cited, appropriate credit is given, any changes made indicated, and the use is non-commercial. See: http://creativecommons.org/licenses/by-nc/4.0/.

\section{ORCID iDs}

Charlotte Hua http://orcid.org/0000-0002-7437-377X

Frank Buttgereit http://orcid.org/0000-0003-2534-550X

Bernard Combe http://orcid.org/0000-0003-4002-1861

\section{REFERENCES}

1 Hoes JN, Jacobs JWG, Buttgereit F, et al. Current view of glucocorticoid co-therapy with DMARDs in rheumatoid arthritis. Nat Rev Rheumatol 2010;6:693-702.

2 Bijlsma JWJ, Van Der Goes MC, Hoes JN, et al. Low-dose glucocorticoid therapy in rheumatoid arthritis: an obligatory therapy. Ann N Y Acad Sci 2010;1193:123-6.

3 Pincus T, Castrejón I, Sokka T. Long-term prednisone in doses of less than $5 \mathrm{mg} /$ day for treatment of rheumatoid arthritis: personal experience over 25 years. Clin Exp Rheumatol 2011;29:S130-8. 
4 Combe B, Landewe R, Daien Cl, et al. 2016 update of the EULAR recommendations for the management of early arthritis. Ann Rheum Dis 2017;76:948-59.

5 Daien $\mathrm{Cl}$, Hua C, Combe B, et al. Non-pharmacological and pharmacological interventions in patients with early arthritis: a systematic literature review Informing the 2016 update of EULAR recommendations for the management of early arthritis. RMD Open 2017;3:e000404.

6 Smolen JS, Landewé R, Bijlsma J, et al. EULAR recommendations for the management of rheumatoid arthritis with synthetic and biological disease-modifying antirheumatic drugs: 2016 update. Ann Rheum Dis 2017;76:960-77.

7 Singh JA, Saag KG, Bridges SL, et al. 2015 American College of rheumatology guideline for the treatment of rheumatoid arthritis. Arthritis Rheumatol 2016;68:1-26.

8 Gaujoux-Viala C, Gossec L. When and for how long should glucocorticoids be used in rheumatoid arthritis? international guidelines and recommendations. Ann N Y Acad Sci 2014;1318:32-40.

9 Black RJ, Lester S, Buchbinder R, et al. Factors associated with oral glucocorticoid use in patients with rheumatoid arthritis: a drug use study from a prospective national biologics registry. Arthritis Res Ther 2017:19.

10 Pincus T, Sokka T, Castrejón I, et al. Decline of mean initial prednisone dosage from 10.3 to $3.6 \mathrm{mg} /$ day to treat rheumatoid arthritis between 1980 and 2004 in one clinical setting, with longterm effectiveness of dosages less than $5 \mathrm{mg} /$ day. Arthritis Care Res (Hoboken) 2013;65:729-36.

11 Makol A, Davis JM, Crowson CS, et al. Time trends in glucocorticoid use in rheumatoid arthritis: results from a population-based inception cohort, 1980-1994 versus 1995-2007. Arthritis Care Res (Hoboken) 2014;66:1482-8.

12 Sokka T, Kautiainen H, Toloza S, et al. QUEST-RA: quantitative clinical assessment of patients with rheumatoid arthritis seen in standard rheumatology care in 15 countries. Ann Rheum Dis 2007:66:1491-6.

13 Albrecht K, Callhoff J, Schneider M, et al. High variability in glucocorticoid starting doses in patients with rheumatoid arthritis: observational data from an early arthritis cohort. Rheumatol Int 2015;35:1377-84.

14 Combe B, Rincheval N. Early lessons from the recentonset rheumatoid arthritis cohort ESPOIR. Joint Bone Spine 2015;82:13-17.

15 Roubille C, Rincheval N, Dougados M, et al. Seven-Year tolerability profile of glucocorticoids use in early rheumatoid arthritis: data from the ESPOIR cohort. Ann Rheum Dis 2017;76:1797-802.

16 McKeown E, Bykerk VP, De Leon F, et al. Quality assurance study of the use of preventative therapies in glucocorticoid-induced osteoporosis in early inflammatory arthritis: results from the catch cohort. Rheumatology 2012;51:1662-9.

17 Chatzidionysiou K, Emamikia S, Nam J, et al. Efficacy of glucocorticoids, conventional and targeted synthetic diseasemodifying antirheumatic drugs: a systematic literature review Informing the 2016 update of the EULAR recommendations for the management of rheumatoid arthritis. Ann Rheum Dis 2017;76:1102-7.

18 Buttgereit F, Mehta D, Kirwan J, et al. Low-Dose prednisone chronotherapy for rheumatoid arthritis: a randomised clinical trial (CAPRA-2). Ann Rheum Dis 2013;72:204-10.

19 Alten R, Grahn A, Holt RJ, et al. Delayed-Release prednisone improves fatigue and health-related quality of life: findings from the CAPRA-2 double-blind randomised study in rheumatoid arthritis. RMD Open 2015;1:e000134.

20 Verschueren P, De Cock D, Corluy L, et al. Patients lacking classical poor prognostic markers might also benefit from a step-down glucocorticoid bridging scheme in early rheumatoid arthritis: week 16 results from the randomized multicenter CareRA trial. Arthritis Res Ther 2015;17.

21 Verschueren P, De Cock D, Corluy L, et al. Effectiveness of methotrexate with step-down glucocorticoid remission induction (cobra slim) versus other intensive treatment strategies for early rheumatoid arthritis in a treat-to-target approach: 1-year results of CareRA, a randomised pragmatic open-label superiority trial. Ann Rheum Dis 2017;76:511-20.

22 Stouten V, Joly J, De Cock D, et al. Sustained Effectiveness after Remission Induction with Methotrexate and Step-Down Glucocorticoids in Patients with Early Rheumatoid Arthritis Following a Treat-to-Target Strategy after 2 Years [abstract]. Arthritis Rheumatol 2017;69(Suppl 10).

23 Markusse IM, Akdemir G, Dirven L, et al. Long-Term outcomes of patients with recent-onset rheumatoid arthritis after 10 years of tight controlled treatment: a randomized trial. Ann Intern Med 2016;164:523-31.

24 Goekoop-Ruiterman YPM, de Vries-Bouwstra JK, Allaart CF, et al. Clinical and radiographic outcomes of four different treatment strategies in patients with early rheumatoid arthritis (the best study): a randomized, controlled trial. Arthritis Rheum 2005;52:3381-90.

25 Safy M, Jacobs J, IJff ND, et al. Long-term outcome is better when a methotrexate-based treatment strategy is combined with $10 \mathrm{Mg}$ prednisone daily: follow-up after the second computer-assisted management in early rheumatoid arthritis trial. Ann Rheum Dis 2017;76:1432-5.

26 Bakker MF, Jacobs JWG, Welsing PMJ, et al. Low-Dose prednisone inclusion in a methotrexate-based, tight control strategy for early rheumatoid arthritis: a randomized trial. Ann Intern Med 2012;156:329-39.

27 Ajeganova S, Svensson B, Hafström I, et al. Low-dose prednisolone treatment of early rheumatoid arthritis and late cardiovascular outcome and survival: 10-year follow-up of a 2-year randomised trial. BMJ Open 2014;4:e004259.

28 Svensson B, Boonen A, Albertsson K, et al. Low-dose prednisolone in addition to the initial disease-modifying antirheumatic drug in patients with early active rheumatoid arthritis reduces joint destruction and increases the remission rate: a two-year randomized trial. Arthritis Rheum 2005;52:3360-70.

29 Hafström I, Albertsson K, Boonen A, et al. Remission achieved after 2 years treatment with low-dose prednisolone in addition to disease-modifying anti-rheumatic drugs in early rheumatoid arthritis is associated with reduced joint destruction still present after 4 years: an open 2-year continuation study. Ann Rheum Dis 2009;68:508-13.

30 Cutolo M. Glucocorticoids and chronotherapy in rheumatoid arthritis. RMD Open 2016;2:e000203.

31 Ursini F, Naty S, Bruno C, et al. Old but good: modified-release prednisone in rheumatoid arthritis. Rev Recent Clin Trials 2017; 12:124-8.

32 Buttgereit F, Strand V, Lee EB, et al. Fosdagrocorat (PF04171327) versus prednisone or placebo in rheumatoid arthritis: a randomised, double-blind, multicentre, phase Ilb study. RMD Open 2019;5:e000889.

33 Spies CM, Straub RH, Cutolo M, et al. Circadian rhythms in rheumatology--a glucocorticoid perspective. Arthritis Res Ther 2014;16(Suppl 2):S3.

34 Kirwan JR, Bijlsma JWJ, Boers M, et al. Effects of glucocorticoids on radiological progression in rheumatoid arthritis. Cochrane Database Syst Rev 2007:CD006356.

35 Graudal N, Jürgens G. Similar effects of disease-modifying antirheumatic drugs, glucocorticoids, and biologic agents on radiographic progression in rheumatoid arthritis: meta-analysis of 70 randomized placebo-controlled or drug-controlled studies, including 112 comparisons. Arthritis Rheum 2010;62:2852-63.

36 van Tuyl LHD, Boers M, Lems WF, et al. Survival, comorbidities and joint damage 11 years after the cobra combination therapy trial in early rheumatoid arthritis. Ann Rheum Dis 2010;69:807-12.

37 Boers M, Verhoeven AC, Markusse HM, et al. Randomised comparison of combined step-down prednisolone, methotrexate and sulphasalazine with sulphasalazine alone in early rheumatoid arthritis. Lancet 1997;350:309-18

38 den Uyl D, ter Wee M, Boers M, et al. A non-inferiority trial of an attenuated combination strategy ('COBRA-light') compared to the original cobra strategy: clinical results after 26 weeks. Ann Rheum Dis 2014;73:1071-8.

39 ter Wee MM, den Uyl D, Boers M, et al. Intensive combination treatment regimens, including prednisolone, are effective in treating patients with early rheumatoid arthritis regardless of additional etanercept: 1-year results of the COBRA-light open-label, randomised, non-inferiority trial. Ann Rheum Dis 2015;74:1233-40.

40 van der Goes MC, Jacobs JWG, Bijlsma JWJ. Rediscovering the therapeutic use of glucocorticoids in rheumatoid arthritis. Curr Opin Rheumatol 2016;28:289-96.

41 Charles-Schoeman C, van der Heijde D, Burmester GR, et al. Effect of glucocorticoids on the clinical and radiographic efficacy of tofacitinib in patients with rheumatoid arthritis: a Posthoc analysis of data from 6 phase III studies. J Rheumatol 2018;45:177-87.

42 Safy M, Jacobs JWG, Edwardes M, et al. No Effect of Concomitant Glucocorticoid Therapy on Efficacy and Safety of Tocilizumab Monotherapy Found in Rheumatoid Arthritis Clinical Trials [abstract]. Arthritis Rheumatol 2018;70(Suppl 10).

43 Choy E, Caporali R, Xavier R, et al. Effects of concomitant glucocorticoids in TOZURA, a common-framework study programme of subcutaneous tocilizumab in rheumatoid arthritis. Rheumatology 2019;58:1056-64. 
44 Bartlett SJ, Bykerk VP, Cooksey R, et al. Feasibility and domain validation of rheumatoid arthritis $(\mathrm{rA})$ flare core domain set: report of the OMERACT 2014 RA flare group plenary. J Rheumatol 2015;42:2185-9.

45 Stenberg VI, Fiechtner JJ, Rice JR, et al. Endocrine control of inflammation: rheumatoid arthritis double-blind, crossover clinical trial. Int J Clin Pharmacol Res 1992;12:11-18.

46 Wolf J, Kapral T, Grisar J, et al. Glucocorticoid treatment in rheumatoid arthritis: low-dose therapy does not reduce responsiveness to higher doses. Clin Exp Rheumatol 2008;26:113-6.

47 Sadra V, Khabbazi A, Kolahi S, et al. Randomized double-blind study of the effect of dexamethasone and methylprednisolone pulse in the control of rheumatoid arthritis flare-up: a preliminary study. Int $J$ Rheum Dis 2014;17:389-93.

48 Burmester GR, Buttgereit F, Bernasconi C, et al. OP0030 randomized controlled 24-week trial evaluating the safety and efficacy of blinded tapering versus continuation of long-term prednisone $(5 \mathrm{Mg} / \mathrm{D})$ in patients with rheumatoid arthritis who achieved low disease activity or remission on tocilizumab. Ann Rheum Dis 2019;78:84-5.

49 Choy EHS, Kingsley GH, Corkill MM, et al. Intramuscular methylprednisolone is superior to pulse oral methylprednisolone during the introduction phase of chrysotherapy. Rheumatology 1993:32:734-9.

50 de Jong PH, Hazes JM, Han HK, et al. Randomised comparison of initial triple DMARD therapy with methotrexate monotherapy in combination with low-dose glucocorticoid bridging therapy; 1-year data of the tREACH trial. Ann Rheum Dis 2014;73:1331-9.

51 Ruyssen-Witrand A, Constantin A. Controversies in rheumatoid arthritis glucocorticoid therapy. Joint Bone Spine 2018;85:417-22.

52 Gvozdenović E, Dirven L, van den Broek M, et al. Intra articular injection with corticosteroids in patients with recent onset rheumatoid arthritis: subanalyses from the best study. Clin Rheumatol 2014;33:263-7.

53 Hetland ML, Østergaard M, Ejbjerg B, et al. Short- and long-term efficacy of intra-articular injections with betamethasone as part of a treat-to-target strategy in early rheumatoid arthritis: impact of joint area, repeated injections, MRI findings, anti-CCP, IgM-RF and CRP. Ann Rheum Dis 2012;71:851-6.

54 Menon N, Kothari SY, Gogna A, et al. Comparison of intra-articular glucocorticoid injections with DMARDs versus DMARDs alone in rheumatoid arthritis. J Assoc Physicians India 2014;62:673-6.
55 Hoes JN, Jacobs JWG, Boers M, et al. EULAR evidence-based recommendations on the management of systemic glucocorticoid therapy in rheumatic diseases. Ann Rheum Dis 2007;66:1560-7.

56 Hoes JN, Jacobs JWG, Verstappen SMM, et al. Adverse events of low- to medium-dose oral glucocorticoids in inflammatory diseases: a meta-analysis. Ann Rheum Dis 2009;68:1833-8.

57 Ravindran V, Rachapalli S, Choy EH. Safety of medium- to longterm glucocorticoid therapy in rheumatoid arthritis: a meta-analysis. Rheumatology 2009;48:807-11.

58 Poppelaars PBM, van Tuyl LHD, Boers M. Normal mortality of the cobra early rheumatoid arthritis trial cohort after 23 years of followup. Ann Rheum Dis 2019;78:586-9.

59 Haraoui B, Jovaisas A, Bensen WG, et al. Use of corticosteroids in patients with rheumatoid arthritis treated with infliximab: treatment implications based on a real-world Canadian population. RMD Open 2015;1:e000078.

60 Santiago T, da Silva JAP. Safety of low- to medium-dose glucocorticoid treatment in rheumatoid arthritis: myths and reality over the years. Ann N Y Acad Sci 2014;1318:41-9.

61 Roubille C, Richer V, Starnino T, et al. The effects of tumour necrosis factor inhibitors, methotrexate, non-steroidal anti-inflammatory drugs and corticosteroids on cardiovascular events in rheumatoid arthritis, psoriasis and psoriatic arthritis: a systematic review and meta-analysis. Ann Rheum Dis 2015;74:480-9.

62 del Rincón I, Battafarano DF, Restrepo JF, et al. Glucocorticoid dose thresholds associated with all-cause and cardiovascular mortality in rheumatoid arthritis. Arthritis Rheumatol 2014;66:264-72.

63 Rau R. Glucocorticoid treatment in rheumatoid arthritis. Expert Opin Pharmacother 2014;15:1575-83.

64 Strehl C, Bijlsma JWJ, de Wit M, et al. Defining conditions where long-term glucocorticoid treatment has an acceptably low level of harm to facilitate implementation of existing recommendations: viewpoints from an EULAR Task force. Ann Rheum Dis 2016;75:952-7.

65 Hartman L, Rasch LA, Klausch T, et al. Harm, benefit and costs associated with low-dose glucocorticoids added to the treatment strategies for rheumatoid arthritis in elderly patients (GLORIA trial): study protocol for a randomised controlled trial. Trials 2018;19:67.

66 Verschueren P, Westhovens R. The use of glucocorticoids in early rheumatoid arthritis. Rheumatology 2018;57:1316-7. 